\title{
A Influência de Vídeos Documentários na Divulgação Científica de Conhecimento sobre a Aids
}

\author{
The Influence of Documentary Videos on the Scientific Diffusion \\ of Knowledge about Aids
}

\author{
Brigido Vizeu Camargo*, Andréa Barbará \& Raquel Bohn Bertoldo** \\ Universidade Federal de Santa Catarina
}

\begin{abstract}
Resumo
O objetivo do estudo foi medir e comparar o impacto sobre o conhecimento científico dos alunos que visualizaram diferentes tipos de vídeo documentário sobre o HIV/Aids - um com abordagem mais científica e outro mais popularizada. Participaram 141 alunos do ensino médio de Florianópolis, divididos em 3 grupos: controle (sem a visualização de vídeo), grupo 1 (vídeo mais científico) e grupo 2 (vídeo mais popularizado). Antes e depois da apresentação dos vídeos foi aplicado o Teste de Conhecimento Científico sobre o HIV/Aids (TCCHA). Os resultados mostraram um aumento no escore médio no TCCHA do grupo 1, nenhuma mudança significativa no grupo 2 e diminuição no grupo controle. Concluiu-se que a forma científica implicou em maior aumento de conhecimento sobre o HIV/Aids.

Palavras-chave: Aids; vídeo informativo; conhecimento científico; adolescentes.

Abstract

The study aimed to measure and to compare the impact on scientific knowledge of students visualizing different kinds of documentary videos about HIV/Aids - one with a more scientific approach and another with a more popularized one. One hundred and forty one (141) high school students from the city of Florianópolis participated in the study. They were divided in 3 groups: control group (without visualization), group 1 (scientific video) and group 2 (popularized video). Before and after the showing the videos, the Scientific Knowledge Test about HIV/Aids was applied. The results showed an increment in the medium score of the mentioned test in group 1, no significant change in group 2 and a decrease in the control group. It was concluded that the scientific form of presentation resulted in an increase of knowledge concerning HIV/Aids.

Keywords: Aids, informative video, scientific knowledge, adolescents.
\end{abstract}

Cresce no país o interesse pela divulgação científica. Centros de ensino e de investigação, publicações - convencionais e eletrônicas, - eventos, entre outros instrumentos, são criados e organizados para divulgar os resultados da ciência junto ao público. A divulgação da ciência é instrumento necessário para consolidar a democracia e evitar que o conhecimento seja sinônimo de poder e dominação. Esta divulgação não pode ser entendida como contribuição para reduzir a ignorância do cidadão, mas um caminho para entender o que ele pensa a respeito de ciência e quais dificuldades têm de avaliar os riscos e valores da vida cotidiana. Ou seja, deve ser um instrumento de comunicação que informe e propicie o diálogo entre os laboratórios e o público (Candotti, 2001; Massarani \& Moreira, 2001). Como ressalta Almeida (2002), “. . . a vida moderna está

\footnotetext{
* Endereço para correspondência: Universidade Federal de Santa Catarina, Centro de Filosofia e Ciências Humanas, Departamento de Psicologia, Campus Universitário Trindade, Florianópolis, SC, 88040-900. Tel.: (48) 3721 9067; Fax: (48) 3721 9984. E-mail: brigido.camargo@yahoo.com.br

** Endereço para correspondência: Universidade Federal de Santa Catarina, Laboratório de Psicologia Social da Comunicação e da Cognição, Trindade, Florianópolis, SC, 88040-900. Tel.: (48) 3331 9067; Fax: (48) 3331 9751. E-mail: raquelbohn@gmail.com
}

cada vez mais dependente da ciência e cada vez mais impregnada dela" (p. 68).

Mas é necessário esclarecer e diferenciar alguns termos e conceitos utilizados na área da divulgação da ciência, tais como: difusão, disseminação, divulgação, popularização e vulgarização. Os termos referem-se a diferentes tipos de público ao qual a atividade é dirigida, a linguagem utilizada e o contexto no qual a informação é divulgada (Bueno, 1985).

Bueno (1985) caracteriza os termos difusão, disseminação e divulgação científica. De acordo com o autor, a "difusão científica" refere-se a todo processo ou recurso utilizado para comunicar informações científicas ou tecnológicas. É uma categoria ampla e global que abarca os outros dois conceitos. Quando essa veiculação dirige-se a especialistas, recebe o nome de "disseminação", e quando é dirigida ao público em geral, denomina-se "divulgação científica".

A disseminação científica “. . . pressupõe a transferência de informações científicas e tecnológicas, transcritas em códigos especializados, a um público seleto, formado por especialistas" (Bueno, 1985, p. 1421).

A divulgação científica “. . . compreende a utilização de recursos, técnicas e processos para veiculação de informa- 
ções científicas e tecnológicas ao público em geral" (Bueno, 1985, p. 1421). Para Abreu (2001), a complexidade da popularização do conhecimento reside na difusão da informação científica sem descontextualizá-la. Neste contexto, vinculam-se livros didáticos, folhetos de campanhas de saúde, documentários, programas especiais de rádio e televisão, jornalismo científico, centros de museus e ciências, revistas em quadrinho, etc. Outro fator importante, além da divulgação impressa, é a rede de informações de acesso eletrônico denominada de internet que, de acordo com Carboni (2005), tem sido um instrumento potente de disseminação e de divulgação da ciência.

Portanto, a divulgação científica consiste em difundir, junto ao grande público, os resultados da pesquisa científica e técnica e, mais freqüentemente, o conjunto das produções do pensamento científico, produzindo mensagens facilmente assimiláveis (Askevis-Leherpeux, Leyens \& Drozda-Senkowska, 2000; Schiele \& Jacobi, 1989).

A divulgação da ciência, conhecida nos Estados Unidos como Science Popularization (Popularização Científica) e na França como Vulgarization Scientifique (Vulgarização Científica) (Mezzomo, 2004), tem sido foco de muitas discussões sobre como tornar o conhecimento científico acessível ao público (Almeida, 2002; Bauer, 1994; Massarani \& Moreira, 2001, 2002). Neste sentido, são necessárias algumas reflexões acerca das possibilidades e limites desta atividade, pois é pertinente que exista um cuidado com a simplificação do conhecimento. Uma crítica à divulgação científica levantada por Barros (2002) é a "vulgarização da ciência" no sentido pejorativo, ou seja, a apresentação da ciência, ou de alguns aspectos que a ciência trabalha, de forma reduzida e banalizada. Segundo o autor, na divulgação da ciência, é comum encontrar o procedimento de "simplificar" para permitir a compreensão por parte de maior número possível de pessoas. A simplificação para tornar acessível "mata" a proposta, e a ciência que é apresentada não tem mais a grandeza nem a profundidade da proposta original.

De acordo com Barros (2002), os programas de difusão científica parecem assumir um novo papel social. Se antes eram atividades que permitiam dar conhecimento para um grupo dominante, hoje os programas de difusão surgem como importantes alternativas para cobrir a defasagem entre o saber escolar e o produzido nos laboratórios e centros de pesquisa.

A circulação de idéias e dos resultados de pesquisas é fundamental para avaliar o seu impacto social e cultural, como também para recuperar, por meio do livre debate e confronto de idéias, os vínculos e os valores culturais que a descoberta do novo, muitas vezes, rompe ou fere (Candotti, 2002). Mas de acordo com Miller (2000), é necessário que a sociedade tenha um nível mínimo de entendimento do processo de ciência e dos termos e conceitos científicos para que possa interagir de maneira consciente na sociedade. $\mathrm{O}$ indivíduo que tem este entendimento desenvolve uma atitude científica frente ao mundo, sendo capaz de interpretar os resultados científicos com base em evidências e poderá nortear seus julgamentos. Reis (2002) ressalta que durante muito tempo a divulgação se limitou a contar ao público os encantos e os aspectos interessantes e revolucionários da ciência e que somente aos poucos passou a refletir, também, a intensidade dos problemas sociais implícitos nessa atividade. A divulgação pela imprensa é muito importante, principalmente em países como o Brasil, onde as dificuldades e as precariedades das escolas fazem com que estudantes e professores obtenham informações sobre os progressos da ciência principalmente por meio de artigos de jornais e da televisão.

Camargo (2003) aponta que a presença dos meios de comunicação social é cada vez mais intensa no modo de vida contemporâneo, especialmente no que se refere à circulação de objetos do debate social, e particularmente em relação à divulgação de informações sobre a epidemia da aids. No Brasil, a epidemia da aids tem seu crescimento desacelerado, entretanto, permanece a tendência ao aumento da incidência entre as mulheres, entre indivíduos de cor parda e por transmissão heterossexual (Ministério da Saúde, 2006).

Os estudos já desenvolvidos focalizaram aspectos comunicacionais da prevenção da aids, como a relação das atitudes e condutas de proteção com o conhecimento adquirido sobre a doença e com as fontes de informação sobre a aids (Camargo, 1997, 1998a; Camargo \& Botelho, 2007; Souza, 2002). Também foram analisadas as representações sociais da aids contidas em uma grande campanha de sensibilização e informação sobre a aids, destinada à televisão francesa e feita sob a forma de concurso de idéias de roteiros de filmes curta metragem (Camargo, 1997, 1998b). Recentemente, um estudo experimental pontuou o impacto de panfletos informativos sobre o conhecimento e as atitudes de adolescentes relativas a aids (Camargo \& Barbará, 2004).

Os resultados de uma das pesquisas, realizada no estado de Santa Catarina (Brasil), com 1.386 estudantes do ensino médio de Florianópolis, Itajaí e Balneário Camboriú, indicaram que persistem falsas crenças sobre a transmissão do HIV, como considerar que o vírus é transmitido em banheiros públicos $(27,2 \%$ dos adolescentes $)$ ou pela picada do mosquito $(22,4 \%)$; bem como aparece confusão quanto à transmissão do HIV pela doação de sangue (34,5\% dos adolescentes) (Camargo \& Botelho, 2007). Em pesquisa com adolescentes do sexo feminino, Romero, Medeiros, Vitalle e Wehba (2007) observaram que embora busquem informações sobre sexualidade, seus conhecimentos sobre DSTs e contracepção são inadequados. Os autores sugerem como solução a implantação de um programa de educação sexual. Percebe-se, portanto, que o conhecimento científico relacionado à DST/Aids circula por meio da mídia e é compartilhado pela população, no entanto, o embasamento do comportamento preventivo consistente torna-se fraco ao mesclar-se a elementos representacionais e culturais.

Embora a televisão seja a mídia mais acessível para todas as camadas da população brasileira (Hamburger, 2001), já foi demonstrado que há relação entre estes problemas de conhecimento sobre a transmissão do HIV e o fato de se ter 
ela como fonte de informação exclusiva (Camargo, 1998a), no entanto, como também acontece nos Estados Unidos, a falta de uma educação sexual consistente, por parte da escola e da família, coloca a televisão como uma das fontes de informação mais utilizada quando se trata de sexualidade (Strasburger, 1999). Hoje, temos uma série de vídeos sobre aids, isto pode ser constatado no Centro de Documentação (CEDOC) da Associação Brasileira Interdisciplinar de aids (ABIA), e uma parcela razoável dos mesmos é destinada aos adolescentes (ver o Web Site: www.abiaids.org.br). Mas a maior parte deles tem uma linguagem prescritiva, voltada para a operacionalização da prevenção. E ainda, duas outras características são encontradas em alguns vídeos: o predomínio de opiniões ou ideologias em detrimento do conhecimento científico (trazendo problemas aos seus conteúdos informacionais) ou a subordinação à linguagem da televisão (enfatizando o espetáculo em detrimento da informação). E estas características resultam na não difusão do conhecimento científico sobre o assunto, e sim sua vulgarização (ver Roqueplo, 1973); o que impede a integração dos avanços científicos à cultura, conforme já observava Jurdant (1975).

Pelas razões já expostas, há pouca divulgação científica em matéria de aids entre nós. E quando há um trabalho similar, ele é feito pela Internet, como o Web Site destinado aos adolescentes (www.adolesite.aids.gov.br) da Coordenação Nacional sobre Doenças Sexualmente Transmissíveis (CN DST/AIDS), ou do Núcleo de Estudos para a prevenção da aids (NEPAIDS) do Instituto de Psicologia da USP (www.usp.br/nepaids), além do Web Site já citado da ABIA. Mas é bom lembrar que, conforme Matsukuma e Leite Filho (2001), nos países em desenvolvimento, a Internet ainda não é um meio acessível a todos.

Supõe-se que um esforço para a divulgação do conhecimento científico acumulado sobre a aids traria benefícios para a prevenção dessa epidemia, na medida o conhecimento mais aprofundado dessa doença pode ter maior impacto que mensagens preventivas de natureza mais sintética.

As observações de Jacquinot em relação ao filme didático (conforme citado por Schiele \& Boucher, 1989), mais precisamente sobre a relação da mensagem do filme com o mundo representado por ela, indicaram que a distinção entre o mundo do especialista e o mundo do amador, no âmbito da construção da mensagem, pode ter um papel importante nesse fluxo descendente de comunicação.

O presente estudo indaga se a visualização de vídeo documentário sobre aids aumentaria o conhecimento anterior sobre a epidemia dos destinatários, na medida em que o vídeo priorizasse mais as informações científicas do que a preocupação em veicular uma narrativa de natureza mais leiga e próxima do público alvo.

\section{Método}

Trata-se de um estudo quase-experimental que avalia o impacto de diferentes tipos vídeo (variável independente) sobre o conhecimento de adolescentes sobre a aids (variável dependente).

\section{Participantes}

Deste estudo, participaram 141 adolescentes, alunos do ensino médio de uma escola pública de Florianópolis. A média de idade da amostra foi de 16 anos e 4 meses $(D P=0,93)$ e esta era formada por $56,03 \%$ participantes do sexo feminino e $43,97 \%$ do sexo masculino.

Inicialmente, foram formados dois grupos: um controle e um experimental, sendo que este último foi subdividido entre aqueles que visualizaram o vídeo 1 (grupo 1) ou o vídeo 2 (grupo 2). O vídeo 1 apresentava uma abordagem mais científica e o vídeo 2 uma abordagem mais popularizada. Assim, os alunos ficaram subdivididos em três grupos: Grupo controle - 56 alunos, Grupo 1 (vídeo científico) - 46 alunos e Grupo 2 (vídeo popularizado) - 39 alunos.

\section{Instrumentos Utilizados}

Foi aplicado um questionário auto-administrado em situação coletiva, composto por questões sobre: (a) variáveis demográficas (idade e sexo); (b) variáveis de interesse para o comportamento sexual (namoro, relações sexuais, uso do preservativo) e (c) questões sobre o vídeo para os grupos experimentais (impressão geral do vídeo, compreensão e conhecimento anterior do conteúdo do vídeo). Nesse questionário foi incluído o Teste de Conhecimento Científico sobre o HIV/ Aids (TCCHA) (Camargo, Barbará \& Bertoldo, 2005).

Teste de Conhecimento Científico sobre a Aids (TCCHA). $\mathrm{O}$ instrumento para a avaliação do conhecimento científico sobre o HIV/Aids é composto por três subtestes: (a) o vírus da aids e sua transmissão (avaliação do conhecimento em relação à aids, ao HIV, a como o HIV ataca o sistema imunológico, à transmissão do HIV, ao teste de detecção do HIV e a distribuição epidemiológica); (b) a infecção pelo HIV e seu tratamento (avaliação do conhecimento em relação à primo-infecção e evolução da aids, aos sintomas menores e forma grave, e ao tratamento da infecção) e (c) prevenção (avaliação do conhecimento em relação à prevenção sexual, aos usuários de droga, à transmissão vertical, à acidentes de trabalho entre profissionais da saúde e a vacinas preventivas) (Camargo et al., 2005). Para ser considerado bem informado cientificamente sobre o HIV/ Aids, o aluno deve atingir um número mínimo de acertos em todos os subtestes: 7 questões no $1^{\circ}$ sub-teste, 4 no $2^{\circ}$ e 6 no $3^{\circ}$.

Vídeos Documentários. Os vídeos apresentados aos alunos foram escolhidos entre aqueles produzidos no Brasil na última década que abordavam conhecimento científico sobre o HIV/Aids. Duas gravações foram selecionadas em função da abordagem do tema e da atualidade do vídeo.

O vídeo 1 (Aids: Série Educação Sexual, 1995¹) apresenta entrevistas com especialistas, portadores e familiares, os quais explicam como se dá a infecção pelo vírus, o

${ }^{1}$ Baldassarini Jr. (1995). 
desenvolvimento da aids e formas de prevenção, enfatizando sempre a inexistência de cura e a importância da doença. O vídeo é rico em dados estatísticos e explicações interativas sobre a síndrome, focando, sobretudo o conhecimento científico.

O vídeo 2 (Amor, vida. Viva! 1990²) é voltado para jovens e adolescentes, com informações básicas sobre o HIV/ Aids, depoimentos de adolescentes portadores do vírus que foram contaminados por meio de transfusão sanguínea, compartilhamento de seringas no uso de drogas injetáveis e de relações sexuais. Também houve a participação de atores e cantores, falando sobre o tema e estimulando o uso do preservativo. Esse vídeo utiliza linguagem e narrativas próprias do contexto adolescente e aborda secundariamente o conhecimento científico sobre a síndrome.

\section{Procedimento}

Primeiramente, foram selecionadas as turmas que iriam participar da pesquisa. Utilizou-se como critério de seleção, o menor contato das turmas com atividades envolvendo aids, ou seja, palestras informativas, atividades de grupo ou pesquisas que envolvessem diretamente o tema da aids. Selecionadas as turmas, foram dividas as que participariam do grupo 1 (vídeo científico), grupo 2 (vídeo popularizado) e controle (sem visualização de vídeo).

No primeiro momento com os grupos, foi aplicado o questionário em situação coletiva. Logo depois, foram apresentados os vídeos nas turmas (vídeo 1 para o grupo 1, vídeo 2 para o grupo 2 e nenhum vídeo para o grupo controle).

Passada uma semana da aplicação do primeiro questionário e dos vídeos, os questionários foram re-aplicados em todos os grupos. Logo depois da re-aplicação, foi feita uma dessensibilização, esclarecidas as dúvidas sobre o assunto e apresentadas as respostas ao TCCHA.

\section{Análise dos Dados}

Os dados do questionário e do Teste de Conhecimento sobre o HIV/Aids envolveram descrição estatística (freqüência relativa, medida de tendência central e dispersão), estatística não paramétrica (teste do Qui-quadrado) e estatística para dados experimentais ( $t$ de Student e ANCOVA). Para isso, foi utilizado o programa informático SPSS (versão 11).

\section{Resultados}

Dos 141 alunos que participaram da pesquisa, 53,3\% tiveram ao menos uma relação sexual com penetração e $40,9 \%$ afirmaram estar namorando no momento da coleta. As médias de idade na primeira coleta eram: 16 anos e 6 meses no grupo controle (grupo que não assistiu a vídeos), 16 anos e 2 meses no grupo 1 (grupo que visualizou o vídeo 1 - conteúdo científico), 16 anos e 3 meses no grupo 2 (grupo que visualizou o vídeo 2 - conteúdo popularizado); não foram encontradas diferenças estatisticamente significativas de idade entre os grupos.

Quanto à experiência sexual dos participantes, no grupo controle $60,7 \%$ já havia tido ao menos uma relação sexual com penetração, $41,3 \%$ no grupo 1 e $51,9 \%$ no grupo 2 . O grupo 1 e o grupo controle apresentaram diferenças significativas quanto à experiência sexual $\left(\chi^{2}=3,81 ; g l=1\right.$; $p<0,05)$ ao passo que os outros grupos não apresentaram diferenças estatisticamente significativas. Como a amostra não é aleatória, é possível que este tipo de diferença tenha certa influência nos resultados do experimento.

$\mathrm{Na}$ re-aplicação dos instrumentos, os alunos puderam opinar sobre o vídeo que haviam assistido. No grupo 1, $60 \%$ dos alunos afirmaram ter gostado do filme e no grupo 2, 74,4\%. Observa-se na Figura 1 que menor percentagem de alunos gostou do vídeo de conteúdo mais científico, no entanto, maior percentagem gostou muito do vídeo 1 .

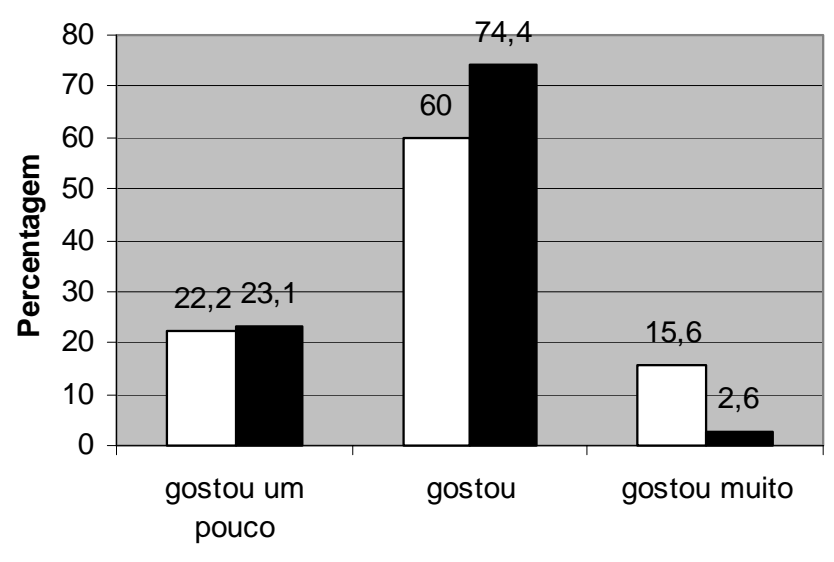

$\square$ Grupo $1 \square$ Grupo 2

Figura 1. Percentagem de alunos que "gostaram um pouco", "gostaram" ou "gostaram muito" por grupo experimental $(N=84)$.

Grande parte $(76,2 \%)$ dos alunos "gostaram" ou "gostaram muito" de ambos os vídeos documentários, o que corrobora Camargo e Botelho (2007), segundo os quais a mídia preferida pelos jovens para a veiculação da informação sobre a aids é o vídeo documentário.

Quanto ao conhecimento prévio das informações veiculadas pelo vídeo, no grupo $1,60 \%$ afirmou conhecer a maior parte das informações e $65,8 \%$ no grupo 2 . Pode-se observar, com base na Figura 2 que foi maior a percentagem de alunos que conheciam a maior parte ou toda informação apresentada pelo vídeo 2 , de conteúdo mais popularizado. Quanto ao vídeo de conteúdo mais científico, 22,2\% conheciam a metade das informações e 2,2\% menos da metade, ou seja, praticamente $1 / 4$ da amostra afirmou conhecer metade, ou menos, das informações apresentadas pelo vídeo 1 . 


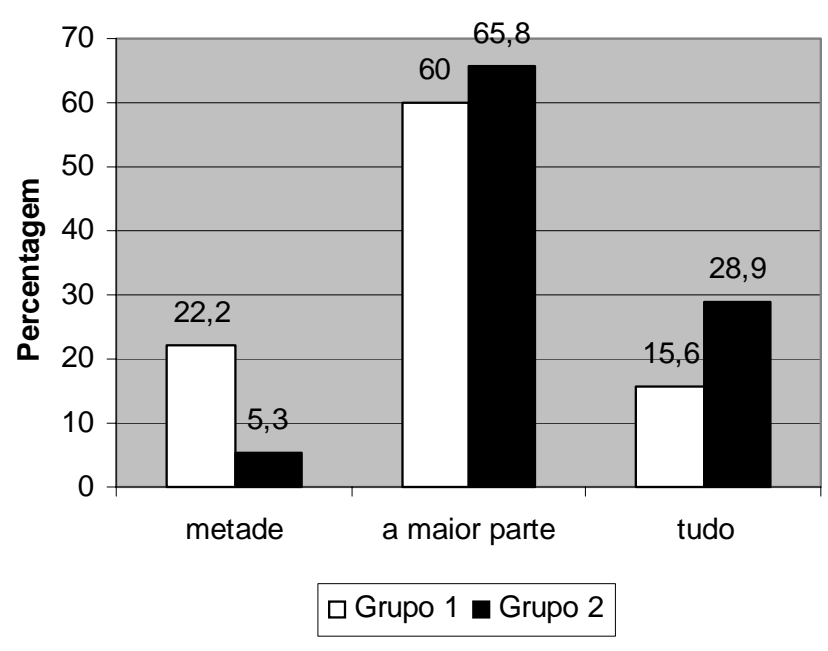

Figura 2. Percentagem da quantidade de conhecimento da informação apresentada nos vídeos por grupo experimental $(N=84)$.

Considerando as Figuras 1 e 2, os grupos experimentais não apresentaram diferenças significativas quanto a gostar pouco, gostar e gostar muito do vídeo, e conhecer metade, a maior parte ou tudo o conteúdo do vídeo pelo número de participantes nos grupos experimentais. Mesmo assim, ressalta-se que o grupo 2 - alunos que assistiram ao vídeo de conteúdo popularizado - conhecia mais sobre a informação apresentada e gostaram mais do vídeo.

Considerando todos os alunos que assistiram aos vídeos, $86 \%$ dos alunos compreenderam tudo ou a maior parte das informações apresentadas no vídeo. No grupo 1, 44,4\% entenderam grande parte e $33,3 \%$, toda a informação; o grupo $2,47,4 \%$ compreenderam grande parte e $50 \%$ compreenderam todo o conteúdo do vídeo. Mesmo que a diferença não tenha sido estatisticamente significativa $\left(\chi^{2}=7,5\right.$; $g l=3 ; P=N$. $S$. , percebe-se que os alunos do grupo 2 compreenderam mais a informação do vídeo a que assistiram que os do grupo 1 .

O conhecimento sobre o HIV/Aids, como já foi descrito acima, também foi medido por meio do Teste de Conhecimento Científico sobre o HIV/Aids (TCCHA). Foram feitas duas medidas - uma antes e outra depois da visualização do vídeo. O escore das medidas iniciais de cada grupo apresentou variações, mas essas diferenças não foram estatisticamente significativas: 15,86 no grupo controle, 15,91 no grupo 1 e 15,72 no grupo 2. Isso significa que quanto ao conhecimento inicial sobre HIV/Aids os grupos não diferiram.

Para controle da variável tempo, o grupo controle foi testado duas vezes quanto ao conhecimento científico sobre HIV/Aids, sem a introdução da variável independente, no espaço de uma semana. Conforme a Figura 3, o impacto da variável tempo no grupo controle representou uma pequena, porém significativa diminuição no escore médio: 15,86 no tempo 1 e 15,08 no tempo $2(t=2,11 ; g l=55 ; p<0,05)$. Segundo Campbell e Stanley (1979), os participantes submetidos a um teste pela segunda vez conseguem usualmente melhores resultados que aqueles que se submetem pela primeira vez. No entanto, a cultura educacional na época em que foi feita essa constatação valorizava tarefas que hoje são consideradas pouco estimulantes, de modo que a diminuição dos escores na segunda aplicação do teste pode ser devida ao cansaço em função de uma atividade já conhecida e, portanto, pouco estimulante.

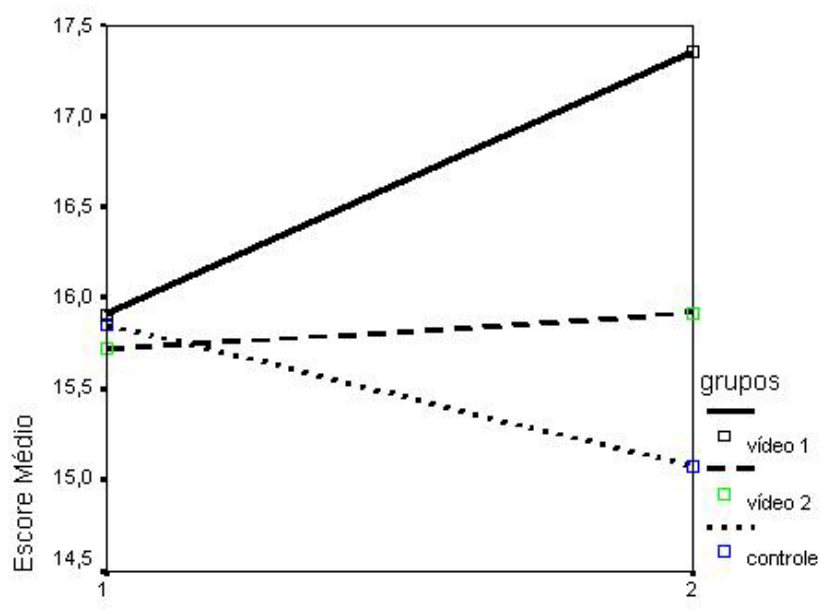

Tempo

Figura 3. Interação do escore médio do TCCHA (tempo 1 e tempo 2) nos grupos (1, 2 e controle) $(N=141)$.

A Figura 3 indica que nos grupos experimentais os vídeos documentários implicaram um acréscimo no escore médio dos grupos. No grupo 1, o escore que no tempo 1 havia sido de 15,91 aumentou para 17,35 no tempo 2 e, no grupo 2 , o escore que no tempo 1 havia sido de 15,72 subiu para 15,92 no tempo 2 , ou seja, um acréscimo de 1,44 e 0,2 respectivamente.

Para avaliar o efeito da variável independente - tipo de vídeo - no conhecimento científico sobre o HIV/Aids TCCHA, foi realizada uma Análise de Covariância (ANCOVA), considerando a segunda medida do TCCHA como variável dependente, e a primeira como covariável nos diferentes grupos. Na primeira análise, a ANCOVA indicou efeito significativo da variável grupo (vídeo 1, vídeo 2 ou controle) na segunda medida do TCCHA $\left(F_{2,140}=8,15\right.$; $p<0,001)$, sendo o tamanho do efeito considerado de moderado para grande $(d$ de Cohen $=0,70)$. O grupo $1-$ que assistiu ao vídeo mais científico - apresentou diferenças estatisticamente significativas do grupo 2 - que assistiu ao filme popularizado (diferença média $=1,3 ; p=0,05$ ) e do grupo controle (diferença média $=2 ; p<0,001$ ). O grupo 2 não apresentou diferenças estatisticamente significativas em relação ao grupo controle.

Além da variável independente grupo, foi medido o efeito da variável sexo na segunda medida do TCCHA que não teve efeito significativo considerado separadamente $\left(F_{1,140}=0,7 ; p=N\right.$. S. $)$ ou em interação com os grupos $\left(F_{2,140}=1,01 ; p=N . S\right.$. $)$. Essa constatação aponta para a independência do sexo para a informação científica, muito embora tenha sido constatado há influência do gênero para 
a retenção de mensagens que contém elementos representacionais e normativos (Camargo \& Barbará, 2004).

$\mathrm{Na}$ análise realizada somente com os grupos experimentais (tipo de vídeo), a variável grupo continua apresentando efeito significativo sobre a segunda medida do TCCHA $\left(F_{1,82}=11 ; p=0,001\right)$, sendo o tamanho do efeito considerado grande $(d$ de Cohen $=0,77)$. Além da variável grupo, a compreensão das informações apresentadas no vídeo teve efeito significativo no conhecimento científico $\left(F_{1,82}=7\right.$; $p=0,01)$, sendo o efeito considerado moderado ( $d$ de Cohen $=0,61)$. Foram medidos também os efeitos de outras variáveis dependentes (o quanto gostou do vídeo, conhecimento anterior das informações) na segunda medida, mas não corresponderam a um efeito estatisticamente significativo.

\section{Discussão}

O experimento pôde evidenciar que somente a apresentação e compreensão do vídeo documentário de conteúdo mais científico, implicaram em aumento do conhecimento sobre o HIV/Aids. A informação científica sobre a aids apresentada de forma mais popularizada, com apelo à emoção e à persuasão por meio da identificação - com o uso de atores e cantores famosos - não implicou em melhoria no conhecimento científico sobre a aids, mesmo que os alunos tenham gostado de ambos. Evidenciou-se que a quantidade de informação veiculada com fins de entretenimento para atrair a atenção dos jovens (no caso, o vídeo 2) não implicou em incremento significativo ao passo que a qualidade científica da informação parece ter incitado a curiosidade dos alunos, gerando impacto significativo na informação científica. No entanto, pelo fato da experiência sexual diferir de modo estatisticamente significativo entre os grupos 1 (que assistiu o vídeo científico) e o controle devese refletir sobre a interferência que este fator poderia ter no impacto do vídeo sobre o conhecimento do grupo 1 .

Campanhas informativas que fazem uso de recursos de identificação ou utilizam valores compartilhados como recurso para atrair a atenção dos jovens, podem suscitar sentimentos de medo, vulnerabilidade frente à aids ou mesmo preconceito contra certos grupos (Camargo, 1997, 2003). Esses elementos normativos, bem como outros problemas relacionados à gestão da racionalidade no contexto sexual, compõem um fenômeno representacional complexo que dificulta a prevenção da doença. Campanhas com enfoque científico, sem apelo emocional e focalizadas nos aspectos objetivos da epidemia consistiriam um dispositivo útil para a divulgação da ciência e, além disso, para o a incorporação de elementos científicos às representações sociais de aids, possibilitando a ação consciente e racionalmente embasada.

As diversas teorias e modelos criados para dar conta da relação entre conhecimento e práticas preventivas continuamente apontam o conhecimento, ou a componente cognitiva como um dos diversos aspectos - percepção do risco no modelo das "crenças sobre a saúde" de Becker; atitudes, comportamentos e normas subjetivas no modelo da "ação refletida" de Fishbein e Ajzen; intenção, hábito, condições facilitadoras e complicadoras no modelo dos "comportamentos interpessoais" de Triandis; e intenções baseadas em informações disponíveis no modelo da "ação racional" de Ajzen (Godin, 1991; Stroebe \& Stroebe, 1995; Zani, 2002). Portanto, o conhecimento científico é parte importante, porém não suficiente, para que o comportamento preventivo seja explicitado.

Tendo em mente o objetivo da popularização do conhecimento científico sem o descaracterizar ou simplificar (Monteiro \& Brandão, 2002), sobretudo em relação à aids e sua repercussão na saúde pública, o presente estudo aponta para o impacto na difusão da informação cientificamente embasada na mídia. A importância da utilização desta última para fins de popularização reside em uma ação cotidiana mais conscientemente fundamentada, com um nível mínimo de entendimento do processo científico para que o indivíduo possa interagir de maneira consciente na sociedade, orientando-se para a prevenção (Miller, 2000).

Como a pesquisa foi realizada com uma amostra escolarizada, não se pode generalizar estes resultados para a população brasileira como um todo. Quanto aos limites do estudo, ressalta-se que no momento da pesquisa havia pouco material áudio-visual atualizado sobre a epidemia da aids, o que limitou as opções de mídia para o experimento. Além disso, o critério para a qualificação e seleção do vídeo de abordagem científica e do de abordagem leiga partiu da equipe de pesquisadores e não de juízes.

\section{Referências}

Abreu, A. R. P. (2001). Estratégias de desenvolvimento científico e tecnológico e a difusão da ciência no Brasil. In S. Crestana, E. W. Hamburger, M. D. Silva \& S. Mascarenhas (Eds.), Educação para a ciência: Curso para treinamento em centros $e$ museus de ciência (pp. 23-28). São Paulo, SP: Livraria da Física.

Almeida, M. O. (2002). A vulgarização do saber. In L. Massarani, I. C. Moreira \& F. Brito (Eds.), Ciência e público: Caminhos da divulgação científica no Brasil (pp. 65-72). Rio de Janeiro, RJ: Centro Cultural de Ciência e Tecnologia da Universidade Federal do Rio de Janeiro.

Askevis-Leherpeux, F., Leyens, J., \& Drozda-Senkowska, E. (2000). Les enjeux ethiques de la diffusion des saviors: l'exemple de la psychology sociale. Bulletin de Psychologie, 53(1), 13-18.

Baldassarini, S., Jr. (Produtor). (1995). Aids: Série Educação Sexual [Vídeo]. São Paulo, SP: SBJ Produções.

Barros, H. L. (2002). A cidade e a ciência. In L. Massarani, I. C. Moreira \& F. Brito (Eds.), Ciência e público: Caminhos da divulgação científica no Brasil (pp. 25-42). Rio de Janeiro, RJ: Centro Cultural de Ciência e Tecnologia da Universidade Federal do Rio de Janeiro.

Bauer, M. (1994). A popularização da ciência como imunização cultural: A função das representações sociais. In S. Jovtchelovitch \& P. Guareschi (Eds.), Textos em representações sociais (pp. 229-257). Petrópolis, RJ: Vozes.

Bueno, W. (1985). Jornalismo científico. Ciência e Cultura, 37(9), 1420-1427. 
Camargo, B. V. (1997). Communication et prévention du sida: études sur le rapport entre l'information télévisuelle, les représentations sociales et la pratique préventive chez les jeunes lycéens. Thèse de doctorat non publiée, Le Programme D'études Supérieures en Psychologie Sociale, École des Hautes Études en Sciences Sociales, Paris.

Camargo, B. V. (1998a). Estudo do conhecimento, das atitudes e dos aspectos de comunicação relativos à prevenção da aids dos estudantes das $3^{\text {as }}$ séries da escola técnica Federal de Santa Catarina ( $1^{\circ}$ semestre de 1997) [Relatório técnico]. Florianópolis, SC: Universidade Federal de Santa Catarina.

Camargo, B. V. (1998b). Representações do preservativo e da aids: Spots publicitários escritos por jovens para a televisão francesa. In D. Jodelet \& M. C. Madeira (Eds.), Aids e representações sociais: À busca de sentidos (pp. 155-173). Natal, RN: Editora da Universidade Federal do Rio Grande do Norte.

Camargo, B. V. (2003). A televisão como vetor de difusão de informações sobre a aids. In M. P. L. Coutinho, A. S. Lima, F. B. Oliveira \& M. L. Fortunato (Eds.), Representações sociais: Abordagem interdisciplinar (pp. 130-152). João Pessoa, PB: Universitária.

Camargo, B., \& Barbará, A. (2004). Efeitos de panfletos informativos sobre a aids em adolescentes. Psicologia: Teoria $e$ Pesquisa, 20(3), 279-287.

Camargo, B. V., Barbará, A., \& Bertoldo, R. (2005). Um instrumento de medida da dimensão informativa da representação social da aids. Trabalho apresentado na IV Jornada Internacional e II Conferência Brasileira sobre Representações Sociais: Teoria e Abordagens Metodológicas, João Pessoa, PB.

Camargo, B. V., \& Botelho, L. J. (2007). Aids, sexualidade e atitudes de adolescentes sobre proteção contra o HIV. Revista de Saúde Pública, 41(1), 61-68.

Campbell, D. T., \& Stanley, J. C. (1979). Delineamentos experimentais e quase-experimentais de pesquisa. São Paulo, SP: Editora da Universidade de São Paulo.

Candotti, E. (2001). Divulgação e democratização da ciência. Ciência e Ambiente, 23(1), 5-14.

Candotti, E. (2002). Ciência na educação popular. In L. Massarani, I. C. Moreira \& F. Brito (Eds.), Ciência e público: Caminhos da divulgação científica no Brasil (pp. 15-24). Rio de Janeiro, RJ: Centro Cultural de Ciência e Tecnologia da Universidade Federal do Rio de Janeiro.

Carboni, L. (2005). A influência de uma exposição científica sobre as representações sociais e atitudes relativas ao meio ambiente: Um estudo com alunos do ensino médio. Dissertação de Mestrado não-publicada, Programa de Pós-Graduação em Psicologia, Universidade Federal de Santa Catarina, Florianópolis, SC.

Godin, H. (1991). L' éducation pour la santé: les fondements psychosociaux de la définition des messages éducatifs. Sciences Sociales et Santé, 9(1), 67-94.

Hamburger, E. W. (2001). A popularização da ciência no Brasil. In S. Crestana, E. W. Hamburger, D. M. Silva \& S. Mascarenhas (Eds.), Educação para a ciência: Curso para treinamento em centros e museus de ciência (pp. 31-40). São Paulo, SP: Livraria da Física.

Jurdant, B. (1975). La vulgarisation scientifique. La Recherche, 6(1), 141-155.

Matsukuma, M., \& Leite, I., Filho (2001). Divulgação científica da estação ciência pela internet. In S. Crestana, E. W. Hamburger, D. M. Silva \& S. Mascarenhas (Eds.), Educação para a ciência: Curso para treinamento em centros e museus de ciência (pp. 475-478). São Paulo, SP: Livraria da Física.
Massarani, L., \& Moreira, I. C. (2001). A retórica e a ciência: Dos artigos originais à divulgação cientifica. Ciência e Ambiente, 23(1), 31-48.

Massarani, L., \& Moreira, I. C. (2002). Aspectos históricos da divulgação científica no Brasil. In L. Massarani, I. C. Moreira \& F. Brito (Eds.), Ciência e público: Caminhos da divulgação científica no Brasil (pp. 43-64). Rio de Janeiro, RJ: Centro Cultural de Ciência e Tecnologia da Universidade Federal do Rio de Janeiro.

Mezzomo, J. (2004). O impacto de uma exposição científica nas representações sociais sobre o meio ambiente dos alunos do ensino médio. Dissertação de Mestrado não-publicada, Programa de Pós-Graduação em Psicologia, Universidade Federal de Santa Catarina, Florianópolis, SC.

Miller, J. (2000). Scientific literacy and citizenship in the $21^{\text {st }}$ century. In B. Schiele \& E. Koster (Eds.), Science centers for this century (pp. 369-413). Quebec, Canada: Multimondes.

Ministério da Saúde (2006). Boletim epidemiológico - AIDS Janeiro a Junho de 2006: Sistema nacional de notificação. Brasília, DF: Coordenação Nacional de DST e AIDS. Retrieved November 30, 2006, from http://www.aids.gov.br/data/

Monteiro, J. R., \& Brandão, S. (2002). Ciência e TV: Um encontro esperado. In L. Massarani, I. C. Moreira \& F. Brito (Eds.), Ciência e público: Caminhos da divulgação científica no Brasil (pp. 89-106). Rio de Janeiro, RJ: Centro Cultural de Ciência e Tecnologia da Universidade Federal do Rio de Janeiro.

Reis, J. (2002). Ponto de vista: José Reis. In L. Massarani, I. C. Moreira \& F. Brito (Eds.), Ciência e público: Caminhos da divulgação científica no Brasil (pp. 73-78). Rio de Janeiro, RJ: Centro Cultural de Ciência e Tecnologia da Universidade Federal do Rio de Janeiro.

Romero, K. T., Medeiros, E. H. G. R., Vitalle, M. S. S., \& Wehba, J. (2007). O conhecimento dos adolescentes sobre questões relacionadas ao sexo. Revista da Associação Médica Brasilei$r a, 53(1), 14-9$.

Roqueplo, P. (1973). Le partage du savoir: science, culture, vulgarisation. Paris: Seuil.

Schiele, B., \& Boucher, L. (1989). L'exposition scientifique: une manière de représenter la science. In D. Jodelet (Ed.), Les représentations sociales (pp. 406-424). Paris: PUF.

Schiele, B., \& Jacobi, D. (1989). La vulgarisation scientifique: théme de recherche. In D. Jacobi \& B. Schile (Eds.), Vulgariser la science (pp. 12-46). Seyssel, France: Vallon.

Souza, E. S. B. (2002). Aspectos comunicacionais e adoção de condutas preventivas, frente ao HIV/AIDS, por adolescentes do ensino médio de Florianópolis, Itajaí e Balneário Camboriú. Dissertação de Mestrado não-publicada, Programa de PósGraduação em Psicologia, Universidade Federal de Santa Catarina, Florianópolis, SC.

Stroebe, W., \& Stroebe, M. S. (1995). Psicologia social e saúde. Lisboa, Portugal: Instituto Piaget.

Strasburger, V. C. (1999). Os adolescentes e a mídia: Impacto psicológico (D. Batista, Trad). Porto Alegre, RS: Artmed.

Teixeira, M., \& Kalili, N. (Diretores). (1990). Amor, vida. Viva! [Vídeo]. Rio de Janeiro, RJ: Associação Brasileira Interdisciplinar de Aids.

Zani, B. (2002). Théories et modèles en psychologie de la santé. In G. N. Fischer (Ed.), Traité de psychologie de la santé (pp. 21-46). Paris: Dunod.

Recebido: 30/11/2006 $1^{a}$ revisão: 04/05/2007 $2^{a}$ revisão: $21 / 08 / 2007$ $3^{a}$ revisão: $30 / 08 / 2007$ Aceite final: 06/09/2007 